\title{
Activation of the CXCL16/CXCR6 Pathway by Inflammation Contributes to Atherosclerosis in Patients with End-stage Renal Disease
}

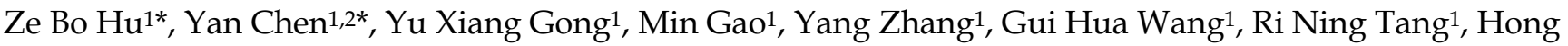 \\ $\mathrm{Liu}^{1}$, Bi Cheng Liu ${ }^{1}$, Kun Ling Ma1, ${ }^{1}$ \\ 1. Institute of Nephrology, Zhongda Hospital, School of Medicine, Southeast University, Nanjing, 210009, China; \\ 2. Department of Nephrology, Taizhou First People's Hospital, Taizhou, 225300, China \\ *The first two authors contributed equally. \\ $\square$ Corresponding author: Kun Ling Ma, Institute of Nephrology, Zhong Da Hospital, School of Medicine, Southeast University, NO.87, Ding Jia Qiao Road, \\ Nanjing City, Jiangsu Province, China, 210009. Tel: 008625 83262442; Email: klma05@163.com \\ () Ivyspring International Publisher. Reproduction is permitted for personal, noncommercial use, provided that the article is in whole, unmodified, and properly cited. See \\ http://ivyspring.com/terms for terms and conditions.
}

Received: 2016.07.04; Accepted: 2016.09.13; Published: 2016.10.20

\begin{abstract}
Background: Chronic inflammation plays a critical role in the progression of atherosclerosis (AS). This study aimed to determine the effects of the CXC chemokine ligand 16 (CXCL16)/CXC chemokine receptor 6 (CXCR6) pathway on cholesterol accumulation in the radial arteries of end-stage renal disease (ESRD) patients with concomitant microinflammation and to further investigate the potential effects of the purinergic receptor P2X ligand-gated ion channel 7 (P2X7R).

Methods: Forty-three ESRD patients were divided into the control group $(n=17)$ and the inflamed group $(n=26)$ based on plasma C-reactive protein (CRP) levels. Biochemical indexes and lipid profiles of the patients were determined. Surgically removed tissues from the radial arteries of patients receiving arteriovenostomy were used for preliminary evaluation of AS. Haematoxylin-eosin (HE) and Filipin staining were performed to assess foam cell formation. CXCL16/CXCR6 pathway-related protein expression, P2X7R protein expression and the expression of monocyte chemotactic protein-1 (MCP-1), tumour necrosis factor- $\alpha$ (TNF- $\alpha$ ), and CD68 were detected by immunohistochemical and immunofluorescence staining.

Results: Inflammation increased both MCP-1 and TNF- $\alpha$ expression and macrophage infiltration in radial arteries. Additionally, foam cell formation significantly increased in the radial arteries of the inflamed group compared to that of the controls. Further analysis showed that protein expression of CXCL16, CXCR6, disintegrin and metalloproteinase-10 (ADAM10) in the radial arteries of the inflamed group was significantly increased. Furthermore, CXCL16 expression was positively correlated with P2X7R expression in the radial arteries of ESRD patients.

Conclusions: Inflammation contributed to foam cell formation in the radial arteries of ESRD patients via activation of the CXCL16/CXCR6 pathway, which may be regulated by P2X7R.
\end{abstract}

Key words: ESRD; inflammation; CXC chemokine ligand 16; purinergic receptor P2X ligand-gated ion channel 7; atherosclerosis

\section{Introduction}

Cardiovascular disease is the most common cause of death for patients with end-stage renal disease (ESRD). ESRD patients have an increased risk of cardiovascular death, 10-20 times that of the general public, and are more likely to die of cardiovascular disease than to progress to dialysis[1]. Atherosclerosis, which is believed to be the common pathophysiological basis of cardiovascular disease, is 
caused by inflammation, oxidative stress, and impaired lipid metabolism ${ }^{2,3]}$. Inflammation and dyslipidemia together accelerate atherosclerosis ${ }^{[4]}$. Ruan et al. ${ }^{[5]}$ confirmed that inflammatory cytokines contribute to foam cell formation by modifying cholesterol-mediated LDL receptor regulation in mesangial cells. However, the mechanisms underlying inflammation-mediated lipid metabolism dysregulation in accelerated atherosclerosis in ESRD are not completely understood.

CXCL16, which was originally described as a scavenger receptor for phosphatidylserine and oxidized low-density lipoprotein (SR-PSOX), is one of the few scavenger receptors that has two distinct forms: membrane-bound and soluble. The membrane-bound form of CXCL16 binds and internalizes oxidative low-density lipoprotein (oxLDL) and promotes adhesion of cells expressing its cognate receptor, CXCR6[6, 7]. In contrast, soluble CXCL16, produced by proteolytic cleavage via ADAM10 and ADAM17[8, 9], acts as a chemotactic factor for CXCR6-expressing cells, such as natural killer T (NKT) cells and polarized T helper cells[10,11]. Wuttge et al. ${ }^{[12]}$ found that the expressions of CXCL16 and CXCR6 were increased in human carotid plaques compared with the normal vein and artery, and interferon- $\gamma$ (IFN- $\gamma$ ) upregulated CXCL16 protein expression both in vivo and in vitro. Gutwein et al.[13] also reported that hyperglycaemic conditions increased CXCL16 and reduced ADAM10 expression, which led to increased uptake of oxLDL in podocytes. These findings suggest that the CXCL16/CXCR6 pathway may contribute to the progression of atherosclerosis in ESRD patients.

P2X purinergic receptors (P2XRs) are plasma membrane cation channels selective for $\mathrm{Na}^{+}, \mathrm{K}^{+}$and $\mathrm{Ca}^{2+}$ that are directly activated by extracellular adenosine triphosphate (ATP) ${ }^{[14]}$. Seven P2X receptor subunits (P2X1-7R) have been identified thus far ${ }^{[14]}$. P2X7R in particular has strong therapeutic potential: this receptor is expressed in cells of the immune system and has a critical role in the normal immune response ${ }^{[15]}$. However, aberrant P2X7R activation contributes to chronic inflammatory disease[16]. Beaucage et al.[17] found that loss of P2X7R increased body and epididymal fat pad weights and reduced total plasma cholesterol levels in mice, suggesting that P2X7R plays a key role in regulating lipid storage and metabolism in vivo. Moreover, Pupovac et al.[18] showed that P2X7R activation induced the rapid shedding of CXCL16. However, the effect of P2X7R activation on lipid metabolism and particularly the modulation of the CXCL16 pathway during chronic inflammation has not been clarified. This study aimed to determine whether inflammation aggravates lipid accumulation in the radial arteries of ESRD patients and to elucidate the possible mechanisms underlying this phenomenon.

\section{Materials and methods}

\section{Ethics Statement}

The study was approved by the Ethical Committee of Taizhou First People's Hospital, and written informed consent was obtained from all subjects.

\section{Patients}

Forty-three ESRD patients from the Blood Purification Centre of Taizhou First People's Hospital were selected for this study between February 2014 and February 2015. ESRD patients receiving haemodialysis treatment were included. Patients with acute infections, cancer and/or chronic active hepatitis were excluded. The patients were divided into two groups according to their plasma C-reactive protein (CRP) levels: a control $(\mathrm{CRP}<3.0 \mathrm{mg} / \mathrm{l})$ and an inflamed group (CRP $\geq 3.0 \mathrm{mg} / \mathrm{l})$.

\section{Clinical biochemical assays}

The body mass index (BMI) and waist circumference (WC) of the ESRD patients were determined. Blood samples were assayed to determine serum levels of CRP, red blood cells (RBCs), haemoglobin $(\mathrm{Hb})$, total protein $(\mathrm{TP})$, albumin (ALB), alanine transaminase (ALT), aspartate transaminase (AST), triglycerides (TGs), total cholesterol (TC), high-density lipoprotein (HDL), low density lipoprotein (LDL), apolipoprotein A1 (Apo A1), Apo B, lipoprotein (a) (Lp(a)), calcium (Ca), phosphate $(\mathrm{P})$, and intact parathyroid hormone (iPTH).

\section{Tissue processing}

Tissues were washed with saline and immediately submerged in $10 \%$ buffered formaldehyde after removal from the radial artery during radial-cephalic anastomosis surgery. After fixation, the tissues were embedded in paraffin.

\section{Haematoxylin and eosin (H \& E) staining}

The paraffin-embedded tissues were sectioned and dewaxed. After washing briefly in distilled water, the sections were stained in Harris haematoxylin solution for 8 minutes, differentiated in $1 \%$ acid alcohol for 30 seconds, and then counterstained in eosin-phloxine solution for 1 minute. The samples were observed with a light microscope $(\times 400)$ after dehydration to transparency and finally sealed with resinene. 


\section{Filipin staining}

After deparaffinization, the sections were rinsed and then stained with Filipin working solution (50 $\mu \mathrm{g} / \mathrm{ml}$ ) for 30 minutes at room temperature. The sections were finally observed by fluorescence microscopy using an ultraviolet filter set package.

\section{Immunohistochemical staining}

After deparaffinization, the sections were placed in excess citrate-buffered solution $(\mathrm{pH}=6.0)$ and microwave until boiling for antigen retrieval. Endogenous peroxidase was blocked with 3\% hydrogen peroxide for 10 minutes at room temperature, and nonspecific antibody binding was blocked with $10 \%$ goat serum. Subsequently, the sections were incubated with goat or rabbit anti-human primary antibodies against tumour necrosis factor a (TNF-a) (Santa Cruz, USA), monocyte chemotactic protein-1 (MCP-1) (Santa Cruz, USA), CXCL16 (R\&D, USA), ADAM10 (Abcam, UK), CXCR6 (NOVUS, USA) and P2X7R (Abcam, UK) overnight at $4^{\circ} \mathrm{C}$, followed by incubation with biotinylated secondary antibodies. Finally, slides were incubated in diaminobenzidine until brown staining was detected. The samples were observed with a light microscope $(\times 400)$.

\section{Immunofluorescence staining}

After deparaffinization, the sections were placed in citrate-buffered solution $(\mathrm{pH}=6.0)$ and then microwaved for antigen retrieval. Subsequently, the sections were incubated with goat or rabbit anti-human primary antibodies against CXCL16, ADAM10, CXCR6, and P2X7R, followed by staining with the fluorescent secondary antibodies donkey anti-goat Alexa Fluor 488 or donkey anti-rabbit Alexa Fluor 594 (Invitrogen, Carlsbad, CA, USA). After washing, the samples were examined by confocal microscopy $(\times 400)$.

\section{Data analysis}

SPSS 16.0 software was used for data analysis. Independent-sample $t$ tests or Mann-Whitney U tests were used for comparison between two groups. The correlation between two groups was determined with Spearman's correlation and Pearson correlation. Differences were considered significant if the $P$ value was less than 0.05 .

\section{Results}

\section{Basic clinical data of the patients in the two groups}

As shown in Table 1, there were no differences in body weight or age of the patients in the two groups. Additionally, there were no differences in the RBC,
Hb, BMI, TP, ALB, TG, TC, HDL, LDL, ApoA1, ApoB, $\mathrm{Ca}, \mathrm{P}$, or iPTH levels $(\mathrm{P}>0.05)$ between the inflamed group and the control (Table 1 ).

Table 1. Basic clinical and biochemical data for the patients

\begin{tabular}{lll}
\hline Parameters & Control $(\mathrm{n}=17)$ & $\begin{array}{l}\text { Inflamed group } \\
(\mathrm{n}=26)\end{array}$ \\
\hline Original disease distribution $(\mathrm{n})$ & \\
CGN & 13 & 13 \\
DN & 2 & 7 \\
HYP & 2 & 6 \\
Weight $(\mathrm{kg})$ & $61.22 \pm 11.00$ & $61.80 \pm 8.59$ \\
BMI $(\mathrm{kg} / \mathrm{m} 2)$ & $22.73 \pm 3.29$ & $22.72 \pm 2.05$ \\
WC (cm) & $79.75 \pm 10.81$ & $81.17 \pm 8.43$ \\
Age (y) & $52.53 \pm 9.40$ & $55.12 \pm 13.33$ \\
RBC $(1012 / \mathrm{L})$ & $2.60 \pm 0.61$ & $2.78 \pm 0.63$ \\
Hb (g/L), Median (IQR) & $78.00(55.50,85.50)$ & $81.00(63.75,92.75)$ \\
TP (g/L) & $62.12 \pm 8.21$ & $60.43 \pm 8.28$ \\
ALB (g/L) & $36.88 \pm 4.25$ & $35.36 \pm 4.16$ \\
ALT (IU/L), Median (IQR) & $13.00(11.00,18.00)$ & $12.00(7.75,23.00)$ \\
AST (IU/L), Median (IQR) & $16.00(12.50,20.00)$ & $17.00(13.00,20.00)$ \\
TG (mmol/L), Median (IQR) & $1.30(0.68,2.10)$ & $1.26(1.00,1.70)$ \\
T-CHO (mmol/L), Median & $3.56(2.75,4.12)$ & $3.75(3.02,4.59)$ \\
(IQR) & & \\
LDL (mmol/L) & $1.81 \pm 0.58$ & $2.07 \pm 0.74$ \\
HDL (mmol/L), Median (IQR) & $1.01(0.81,1.21)$ & $1.06(0.81,1.25)$ \\
ApoA1 (mmol/L), Median & $1.13(1.03,1.28)$ & $1.12(0.96,1.38)$ \\
(IQR) & & \\
ApoB (mmol/L) & $0.71 \pm 0.22$ & $0.78 \pm 0.23$ \\
Lp(a) (mmol/L), Median & $207.00(134.00,324.00)$ & $249.00(154.00,415.00)$ \\
(IQR) & & \\
Ca (mmol/L) & $2.10 \pm 0.24$ & $2.00 \pm 0.25$ \\
P (mmol/L) & $2.08 \pm 0.53$ & $1.96 \pm 0.74$ \\
Ca $\times$ P (mmol/L) & $54.41 \pm 11.26$ & $50.13 \pm 19.58$ \\
iPTH (pg/mL), Median (IQR) & $335.30(194.05,854.70)$ & $323.00(144.32,467.30)$ \\
\hline Abbreviation: IQR, interquartile range. CGN = chronic glomerulonephritis; DN $=$ \\
diabetic nephropathy; HYP = hypertension. There was no difference in every index \\
in the inflamed group compared with that in the control, P>0.05.
\end{tabular}

\section{Inflammation increased inflammatory cytokine expression and macrophage infiltration}

As shown in Figure 1, inflammation increased protein expressions of both MCP-1 and TNF- $\alpha$ in the radial arteries of the inflamed group, along with increased macrophage infiltration. These results suggest that local inflammation of the radial arteries is induced in the inflamed group, which is consistent with the observation of systemic inflammation.

\section{Inflammation induced foam cell formation in the radial arteries}

To evaluate the effect of inflammation on the progression of atherosclerosis, we assessed foam cell formation by HE staining and cholesterol accumulation by Filipin staining. There was significant foam cell formation in the radial arteries of the inflamed group compared with that of the control group (Fig. 2A, 2B), and it was predominantly found in the middle muscle tissues of the vessels. Filipin staining showed that cholesterol accumulation in the 
radial arteries of the inflamed group was increased (Fig. 2C).

\section{Inflammation increased protein expressions of the CXCL16 pathway in the radial arteries}

To explore the potential mechanisms of foam cell formation induced by inflammation, we evaluated the effects of inflammation on the protein expression of the CXCL16 pathway by immunohistochemical and immunofluorescence staining in the radial arteries. As shown in Fig. 3, inflammation significantly increased protein expressions of CXCL16, ADAM10, and CXCR6 in the radial arteries of ESRD patients (Fig. 3A-3D). Moreover, the plasma CRP level was positively correlated with the expression of the CXCL16 protein (Fig. 3E, $\mathrm{R}=0.824, \mathrm{P}<0.05$ ).

A

TNF- $\alpha$
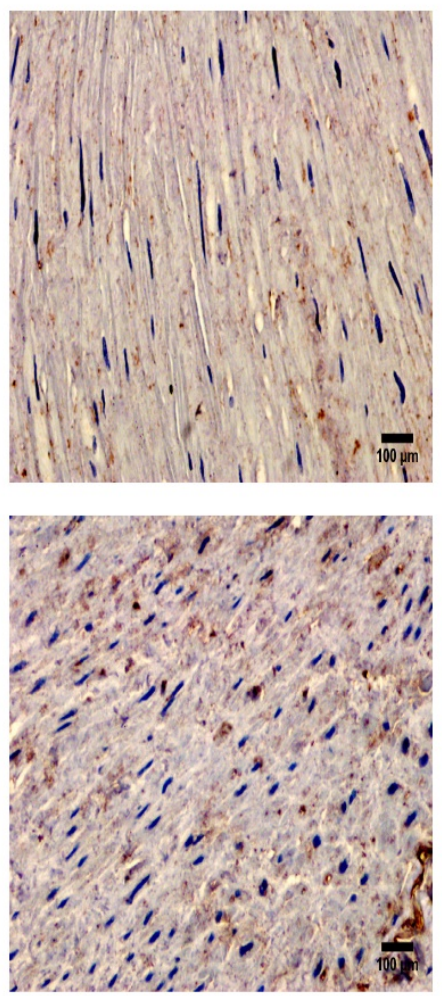

MCP-1
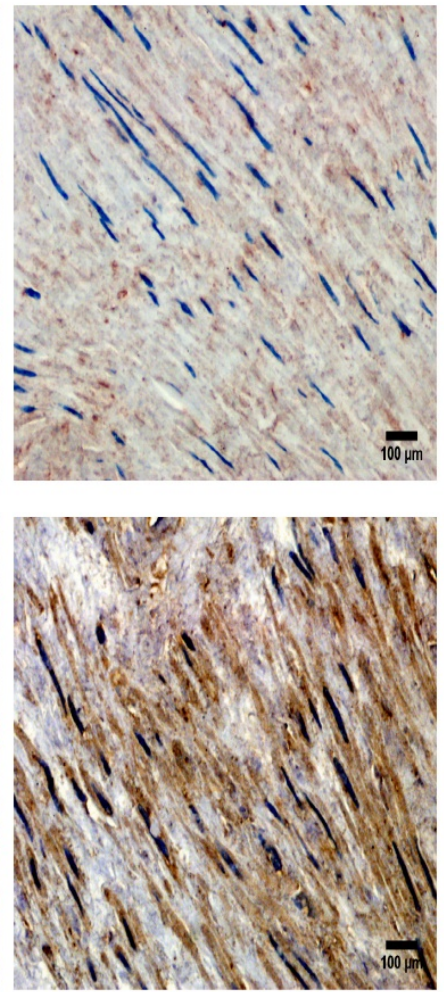

CD68
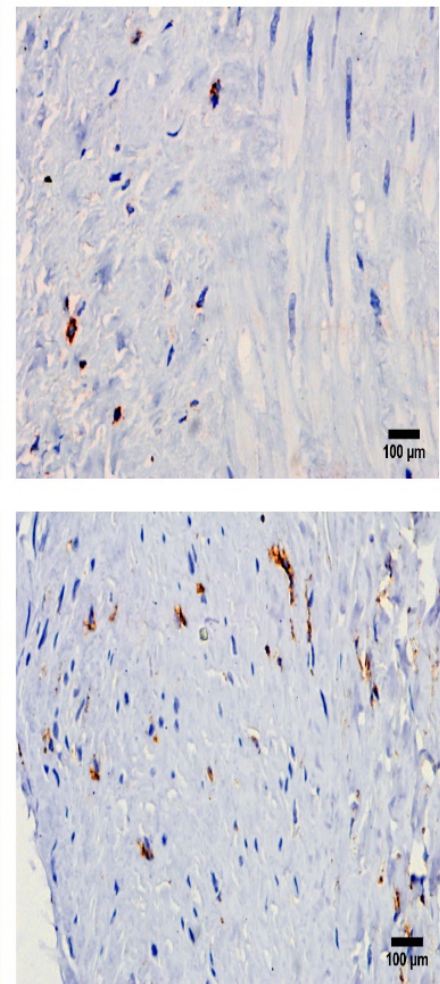

B

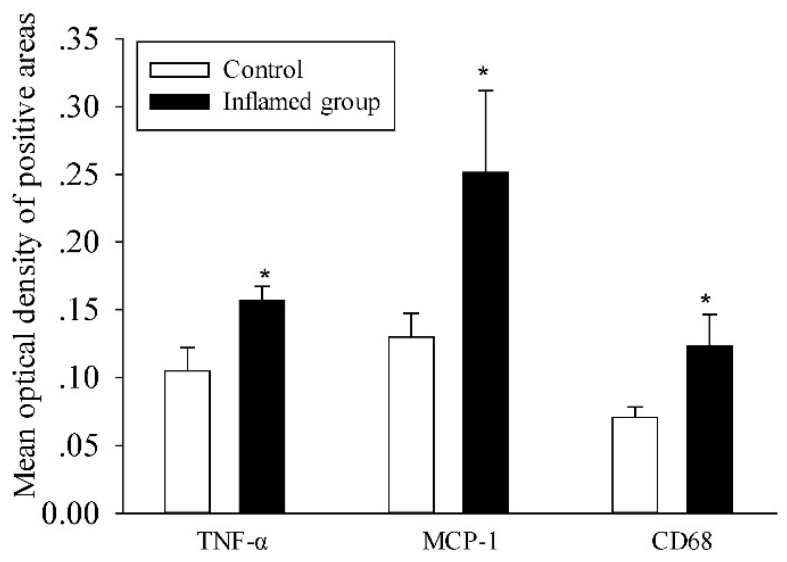

Fig. I Inflammation increased inflammatory cytokine expression and macrophage infiltration. TNF- $\alpha$, MCP-1, and CD68 protein expressions in the radial arteries were examined by immunohistochemical staining. (A, brown colour, original magnification $\times 400)$. The values of semiquantitative analysis of the positive areas are expressed as the mean \pm SD from five patients in each group ( $n=17$ for control, $n=26$ for inflamed group). $* P<0.05$ vs control (B). 
A

Control

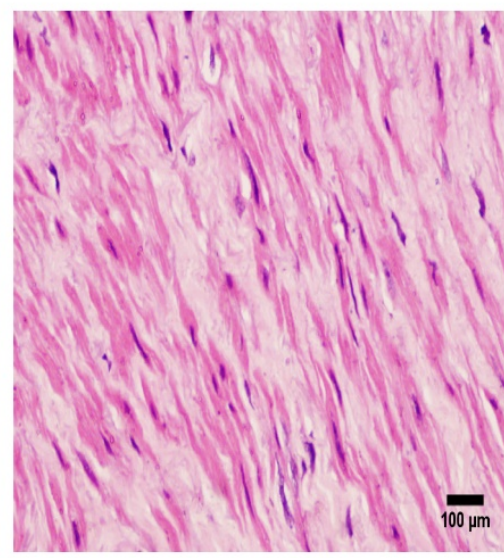

Control

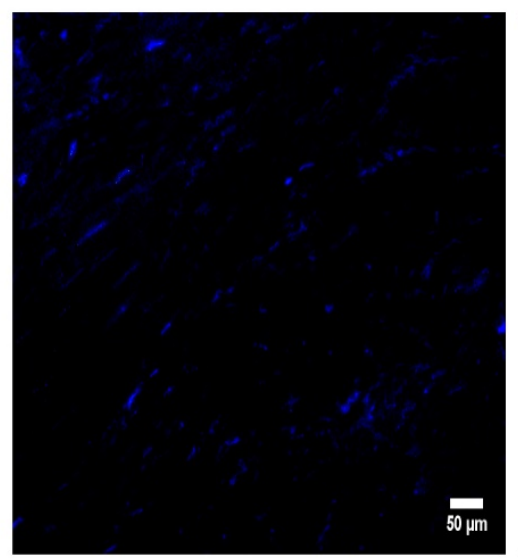

C
Inflamed group

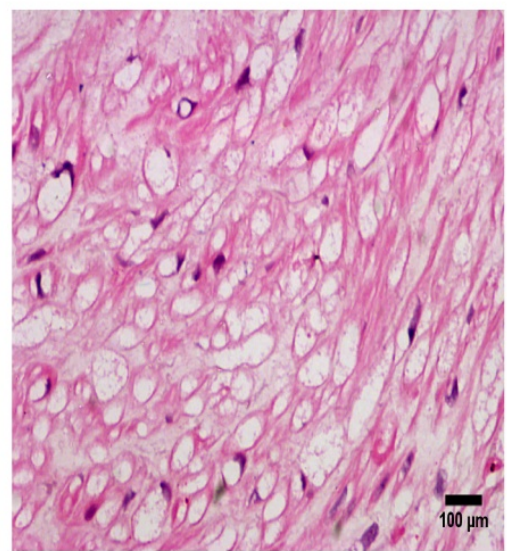

Inflamed group

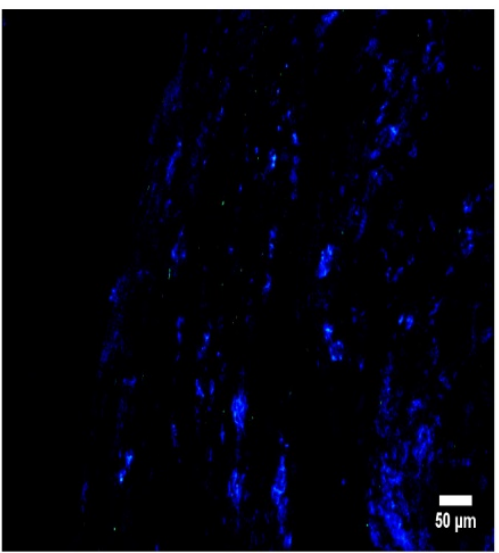

$10 \mu \mathrm{m}$

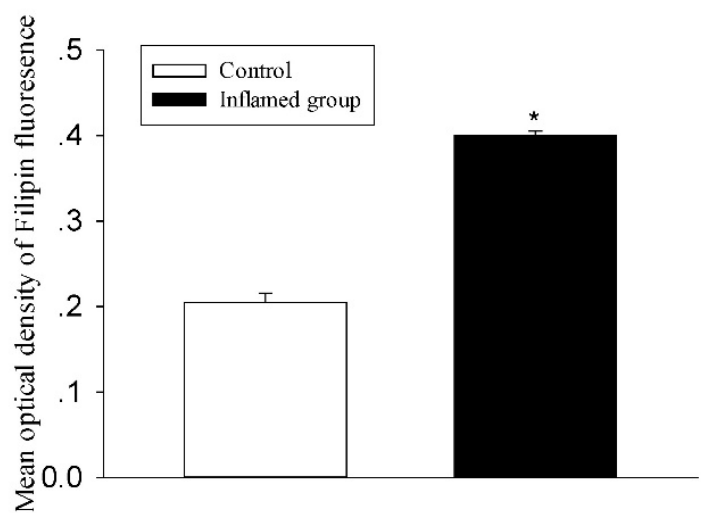

Fig. 2 Inflammation induced foam cell formation and increased cholesterol accumulation in the radial arteries. The lipid accumulation in the radial arteries was assessed by haematoxylin-eosin staining $(\mathbf{A}$, original magnification $\times 400)$ and Filipin staining (B, original magnification $\times 200)$. The values of semiquantitative analysis of the positive areas are expressed as the mean \pm SD from five patients in each group ( $n=17$ for control, $n=26$ for inflamed group). $* P<0.05$ vs. control (C). 
A

\section{CXCL16}

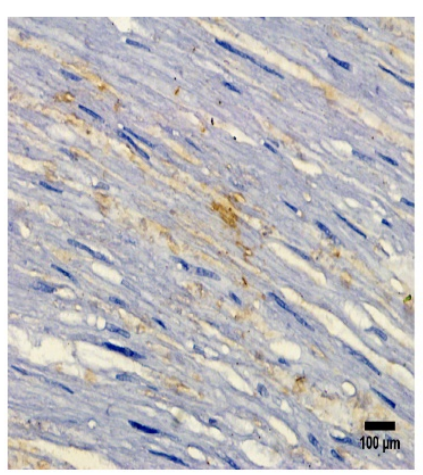

Control

Inflamed group
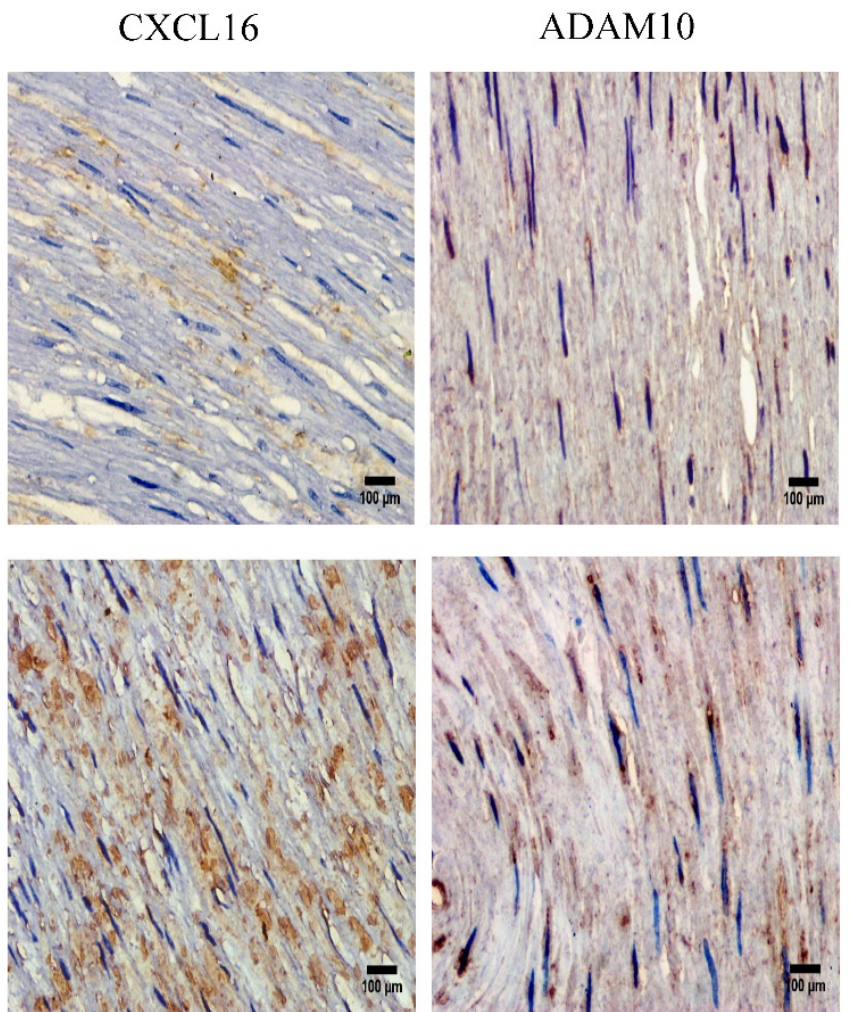

\section{CXCR6}
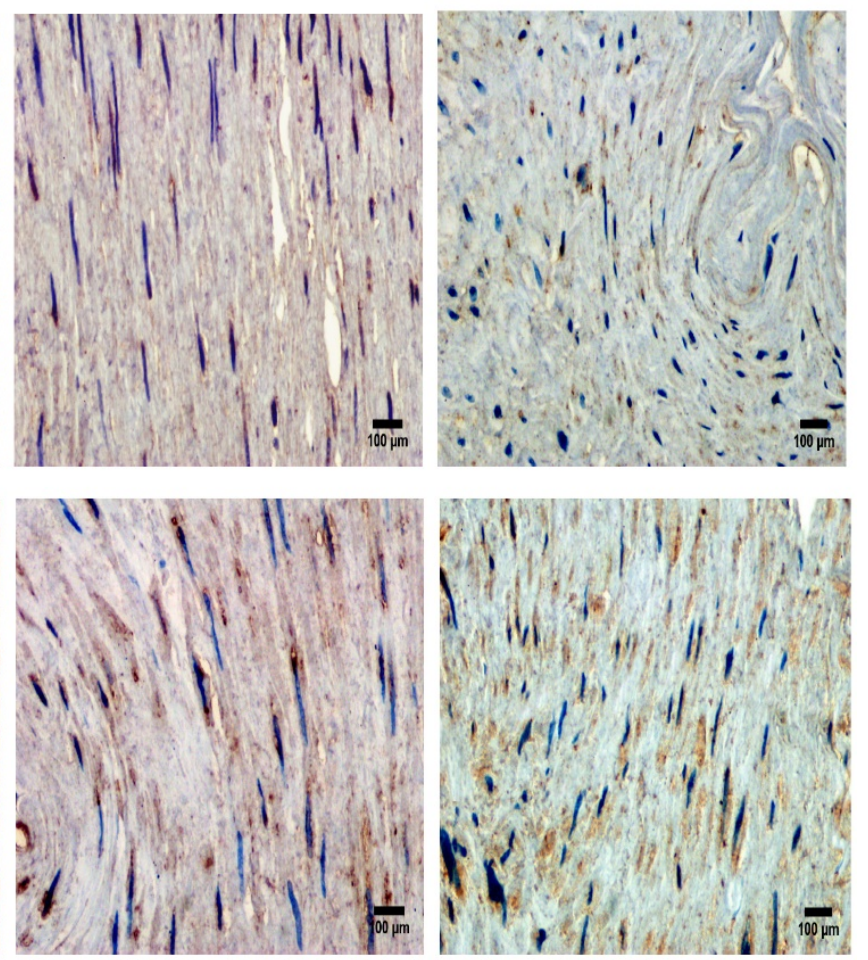

B

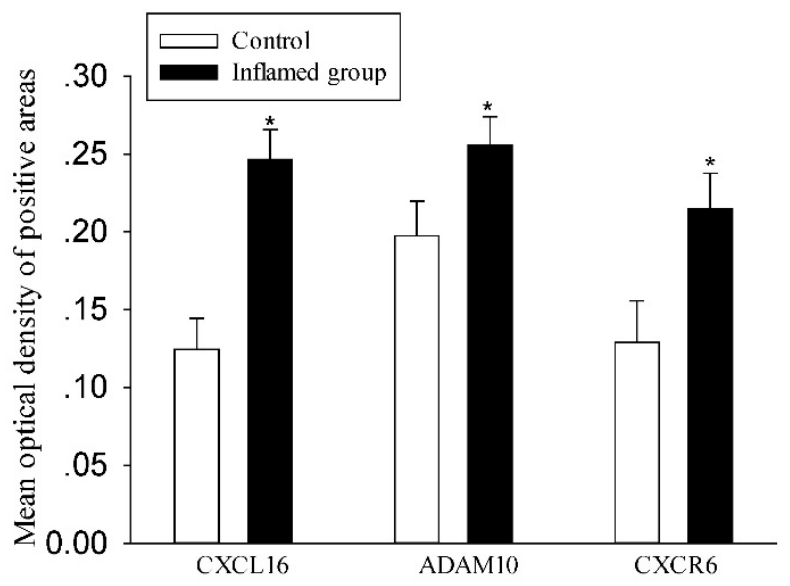


C

Control

Inflamed group
CXCL16
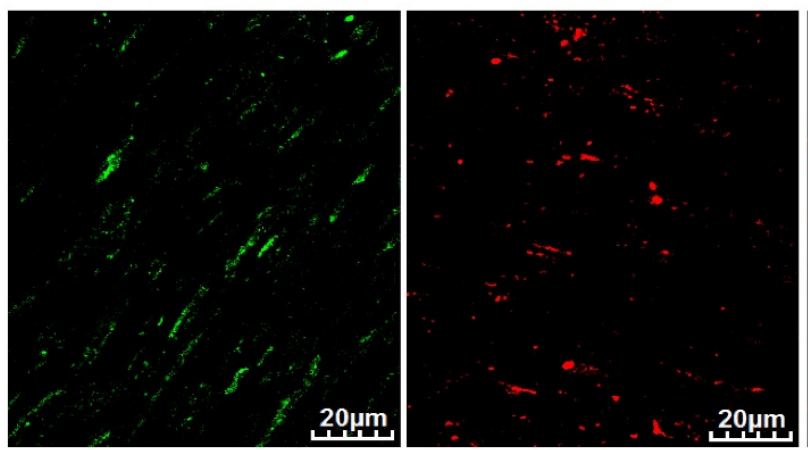

ADAM10

CXCR6
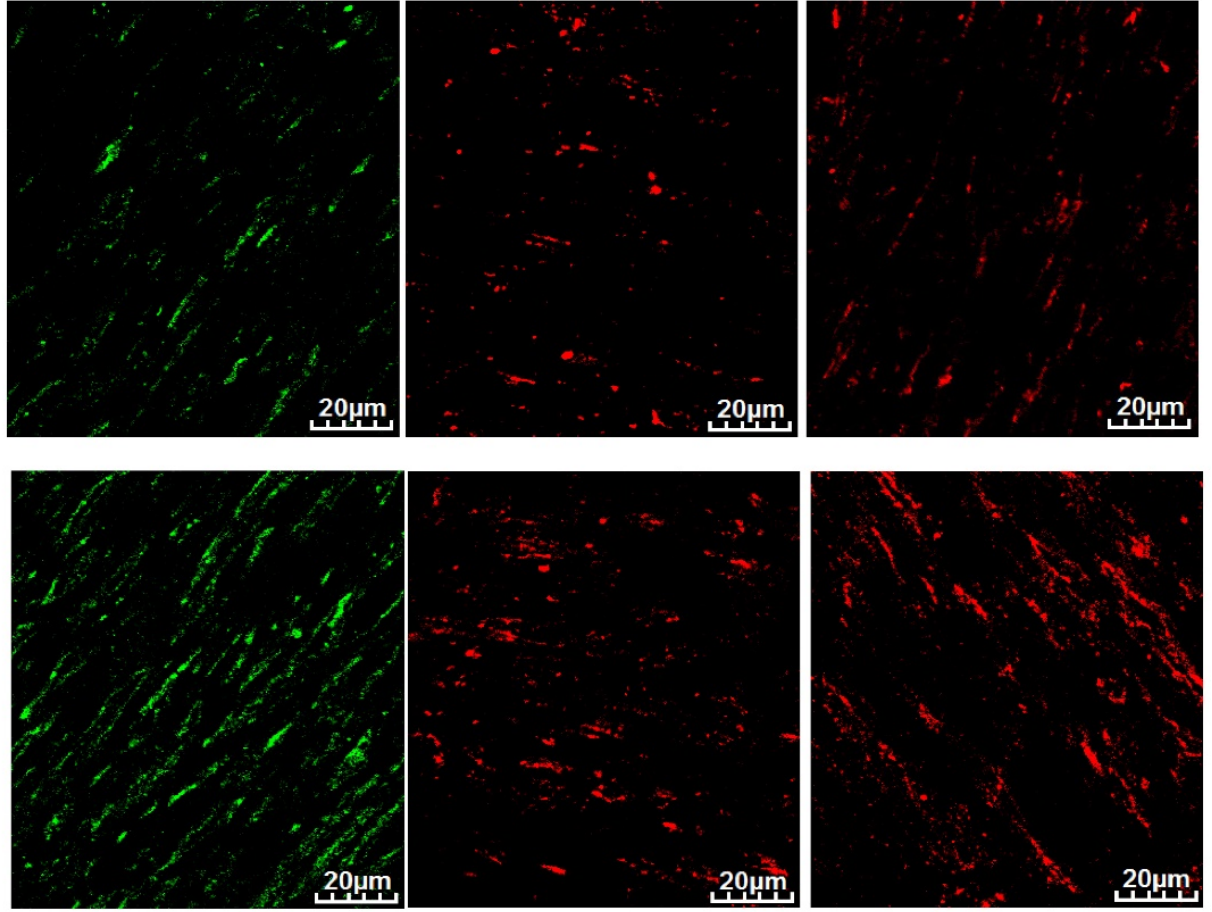

D

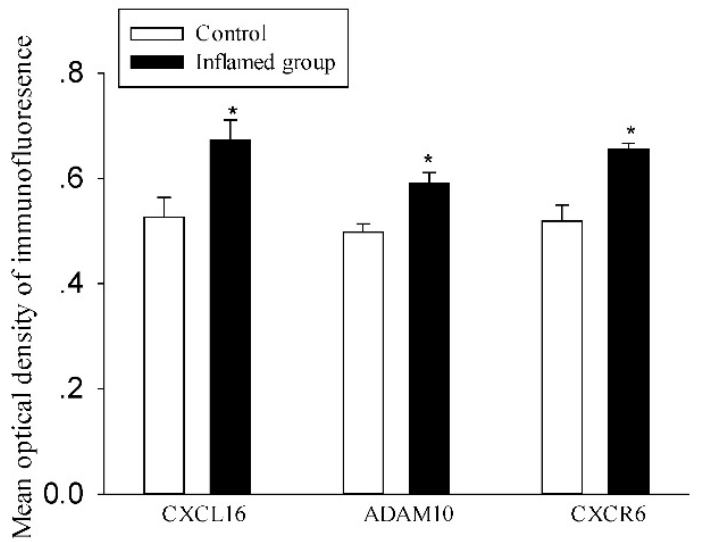

$\mathbf{E}$

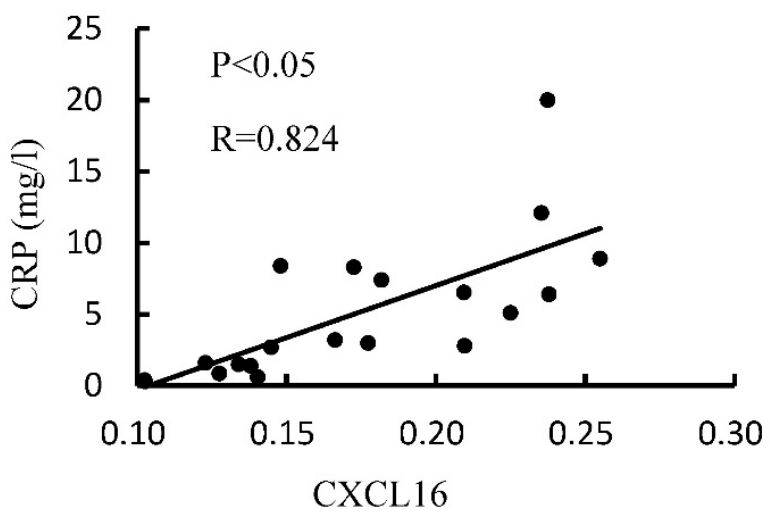

Fig. 3 Inflammation increased CXCL16 pathway protein expression in the radial arteries. The protein expressions of CXCL16, ADAM10 and CXCR6 in the radial arteries were measured by immunohistochemical staining $(\mathbf{A}$, brown colour, original magnification $\times 400)$ and immunofluorescence staining $(\mathbf{C}$, original magnification $\times 400$ ). The values of semiquantitative analysis of the positive areas are expressed as the mean \pm SD from five patients in each group ( $n=17$ for control, $\mathrm{n}=26$ for inflamed group). $* \mathrm{P}<0.05$ vs control (B, D). Correlation analysis of plasma CRP level with CXCL16 expression (E). 
Increased protein expressions of the CXCL16 pathway were positively correlated with increased $P 2 X 7 R$ expression in the radial arteries

Activation of the purinergic P2X7R has been shown to induce the rapid shedding of CXCL16. Therefore, we evaluated the association between the activation of P2X7R and the CXCL16 pathway. Using immunohistochemical and immunofluorescence staining, we observed that inflammation also significantly increased protein expression of P2X7R (Fig. 4A-4D). To investigate the relationship between CXCL16 and P2X7R expression in the radial arteries, we evaluated the immunohistochemical expressions of the two proteins using Image-Pro Plus software. Spearman's correlation analysis of CXCL16 expression $v s$. P2X7R revealed a positive correlation (Fig. 4E, $\mathrm{R}=0.610, \mathrm{P}<0.05$ ).
A

P2X7R
Control

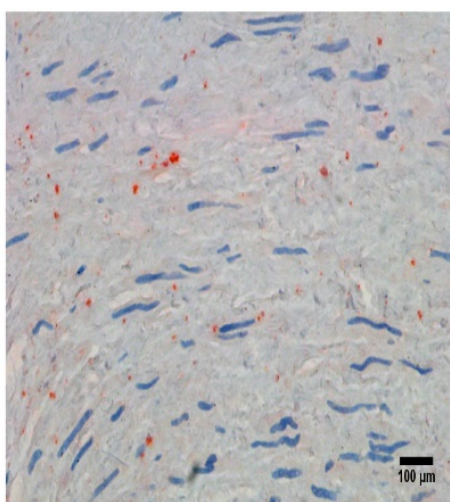

Inflamed group

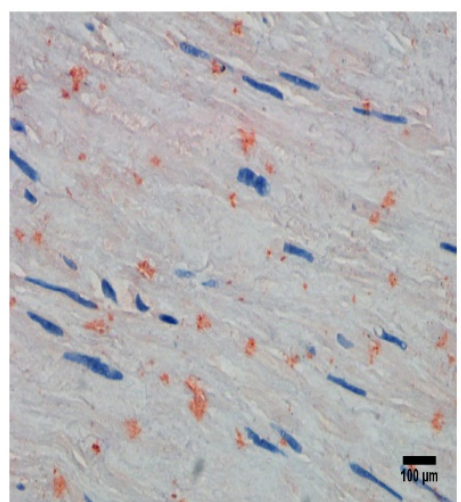

B

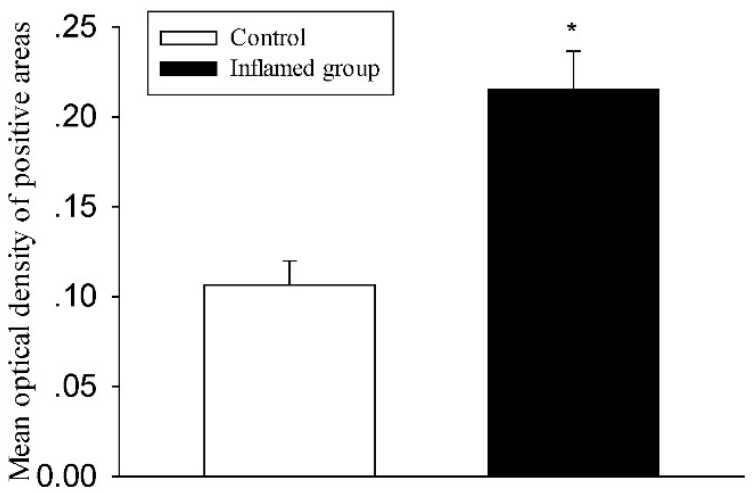

C

Control

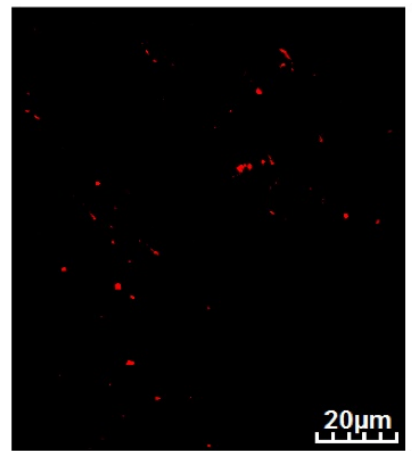

Inflamed group

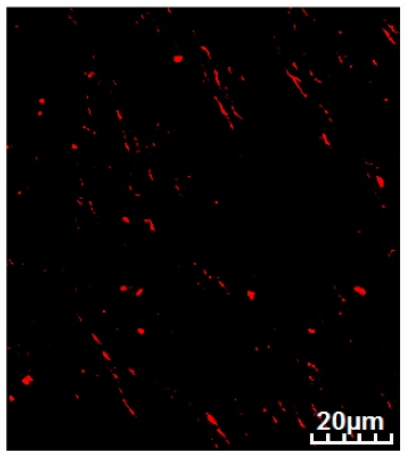




\section{D}
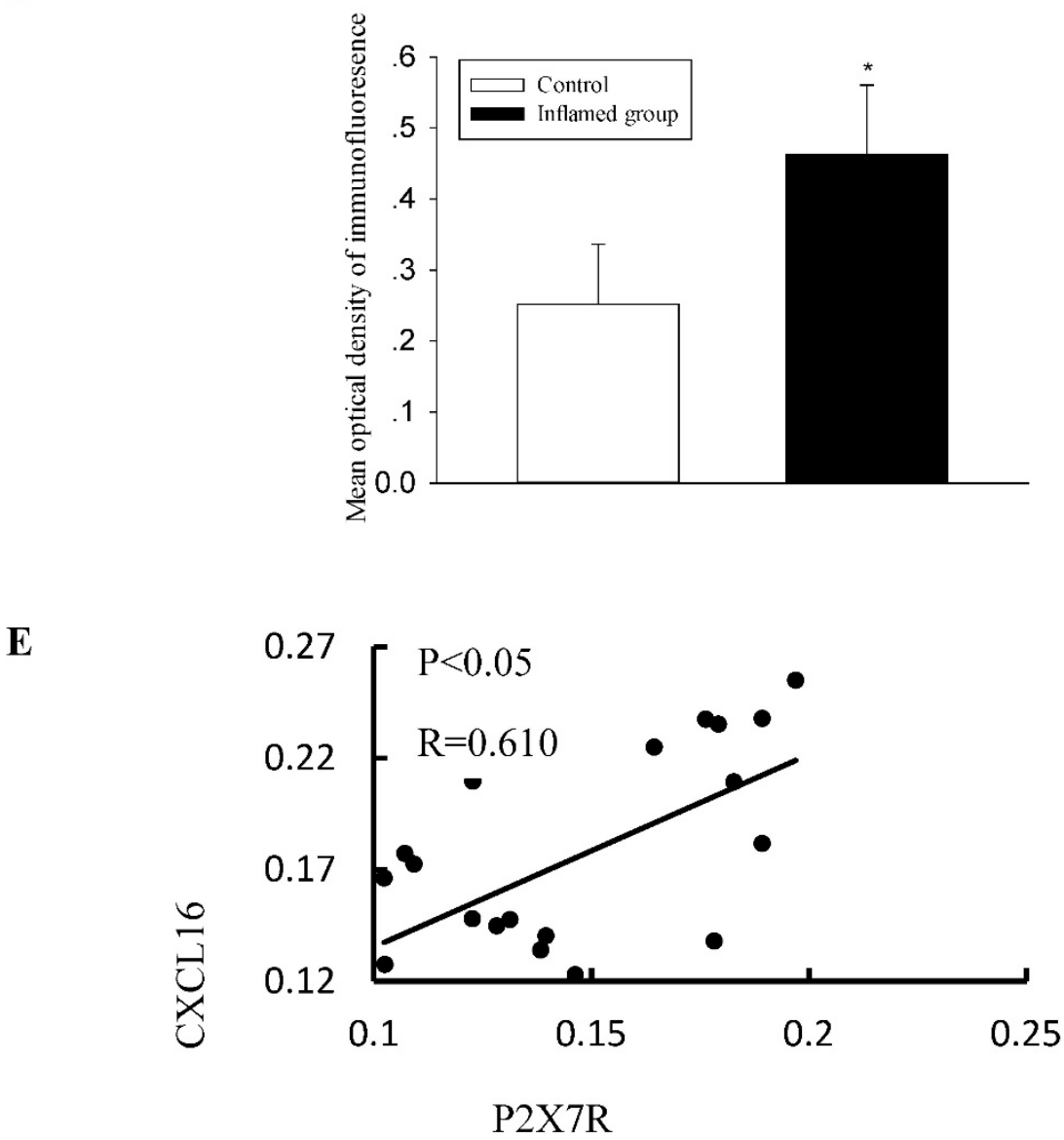

Fig. 4 Increased CXCL16 pathway protein expression was positively correlated with P2X7R expression in the radial arteries. The protein expression of P2X7R in the radial arteries was measured by immunohistochemical staining (A, brown colour, original magnification $\times 400)$ and immunofluorescence staining (C, original magnification $\times 400$ ). The values of semiquantitative analysis of the positive areas are expressed as the mean \pm SD from five patients in each group ( $n=17$ for control, $n=26$ for inflamed group). * $P<0.05$ vs control (B, D). Correlation analysis was performed between CXCL16 expression and P2X7R expression (E).

\section{Discussion}

Chronic inflammation and atherosclerosis are common features in ESRD patients. More recently, atherosclerosis has been shown to be an inflammatory disease as well as a lipid disorder. Our previous studies demonstrated that inflammation induced intracellular lipid accumulation and foam cell formation by disrupting LDL receptor feedback regulation, contributing to the progression of atherosclerosis ${ }^{[19-21]}$. In this study, we investigated the role of CXCL16, which acts as a scavenger receptor and chemokine in different forms, in the progression of atherosclerosis in ESRD patients under inflammatory stress.

We found that inflammation significantly increased TNF- $\alpha$ and MCP-1 protein expressions in the arteries along with increased cholesterol accumulation and foam cell formation, which was consistent with our previous studies ${ }^{[2]}$. This report provided further clinical evidence that inflammation contributes to the progression of atherosclerosis in ESRD patients.

It has been reported that CXCL16 was expressed in both human and murine atherosclerotic lesions and promoted atherogenesis ${ }^{[12]}$. Galkina et al. ${ }^{[23]}$ also found that CXCR6-deficient apolipoprotein E knockout mice had attenuated atherosclerosis. However, Aslanian and Charo observed accelerated atherosclerosis in CXCL16-null LDL receptor-/- mice[24]. Aslanian et al.[25] suggested that a possible explanation for these inconsistent observations may be that CXCL16, which has both chemoattractant and scavenger receptor functions, predominantly performs one of the functions and inhibits the other one. In our study, we found that CXCL16 pathway-related protein expression was significantly upregulated by inflammation, indicating that the CXCL16 pathway may contribute to foam cell formation in the arteries of ESRD patients.

Pupovac et al. ${ }^{[18]}$ reported that P2X7R may be involved in the regulation of CXCL16. Our results also 
showed that P2X7R expression was significantly increased in the inflamed group compared with that of the controls. Further analysis showed that there was a positive correlation between the expressions of CXCL16 and P2X7R in the arteries of ESRD patients. This suggests that the effect of the CXCL16 pathway on foam cell formation during chronic inflammation may be correlated with P2X7R activation. Piscopiello et al.[26] first reported expression of P2X7R on human vessels and suggested a role for P2X7R in atherosclerosis. Furthermore, Peng et al.[27] recently showed that P2X7R exacerbated atherosclerosis by promoting NLRP3 inflammasome activation. Therefore, P2X7R is believed to participate in lipid accumulation primarily through its inflammatory phenotype. Our study indicated that P2X7R may be involved in lipid metabolism dysfunction by modulating the CXCL16 pathway.

In conclusion, our findings are the first to demonstrate that inflammation contributes to the development of atherosclerosis in ESRD patients via the upregulation of the CXCL16 pathway, which was correlated with P2X7R activation. These observations may improve our understanding of the mechanism of atherosclerosis in ESRD.

\section{Acknowledgements}

This work was supported by the Jiangsu Province Ordinary University Graduate Research Innovation Project (KYZZ15-0061), the National Natural Science Foundation of China (grants 81170792 and 81470957), the Natural Science Foundation of Jiangsu Province (BK20141343), and the Clinical Medical Science Technology Special Project of Jiangsu Province (BL2014080).

\section{Competing Interests}

The authors have declared that no competing interest exists.

\section{References}

[1] Foley RN, Parfrey PS, Sarnak MJ. Clinical epidemiology of cardiovascular disease in chronic renal disease. Am J Kidney Dis. 1998. 32(5 Suppl 3): S112-9.

[2] Siti HN, Kamisah Y, Kamsiah J. The role of oxidative stress, antioxidants and vascular inflammation in cardiovascular disease (a review). Vascul Pharmacol. 2015. 71: 40-56.

[3] Keane WF, Tomassini JE, Neff DR. Lipid abnormalities in patients with chronic kidney disease: implications for the pathophysiology of atherosclerosis. J Atheroscler Thromb. 2013. 20(2): 123-33.

[4] Steinberg D. Atherogenesis in perspective: hypercholesterolemia and inflammation as partners in crime. Nat Med. 2002. 8(11): 1211-7.

[5] Ruan XZ, Varghese Z, Powis SH, Moorhead JF. Dysregulation of LDL receptor under the influence of inflammatory cytokines: a new pathway for foam cell formation. Kidney Int. 2001. 60(5): 1716-25.

[6] Shimaoka T, Kume N, Minami M, Hayashida K, Kataoka H, Kita T et al. Molecular cloning of a novel scavenger receptor for oxidized low density lipoprotein, SR-PSOX, on macrophages. J Biol Chem. 2000. 275(52): 40663-6.

[7] Shimaoka T, Nakayama T, Fukumoto N, Kume N, Takahashi S, Yamaguchi J et al. Cell surface-anchored SR-PSOX/CXC chemokine ligand 16 mediates firm adhesion of CXC chemokine receptor 6-expressing cells. J Leukoc Biol. 2004. 75(2): 267-74
[8] Abel S, Hundhausen C, Mentlein R, Schulte A, Berkhout TA, Broadway N et al. The transmembrane CXC-chemokine ligand 16 is induced by IFN-gamma and TNF-alpha and shed by the activity of the disintegrin-like metalloproteinase ADAM10. J Immunol. 2004. 172(10): 6362-72.

[9] Gough PJ, Garton KJ, Wille PT, Rychlewski M, Dempsey PJ, Raines EW. A disintegrin and metalloproteinase 10-mediated cleavage and shedding regulates the cell surface expression of CXC chemokine ligand 16. J Immunol. 2004. 172(6): 3678-85.

[10] Geissmann F, Cameron TO, Sidobre S, Manlongat N, Kronenberg M, Briskin MJet al. Intravascular immune surveillance by CXCR6+ NKT cells patrolling liver sinusoids. PLoS Biol. 2005. 3(4): e113.

[11] Matloubian M, David A, Engel S, Ryan JE, Cyster JG. A transmembrane CXC chemokine is a ligand for HIV-coreceptor Bonzo. Nat Immunol. 2000. 1(4): 298-304.

[12] Wuttge DM, Zhou X, Sheikine Y, Wagsater D, Stemme V, Hedin U et al. CXCL16/SR-PSOX is an interferon-gamma-regulated chemokine and scavenger receptor expressed in atherosclerotic lesions. Arterioscler Thromb Vasc Biol. 2004. 24(4): 750-5.

[13] Gutwein P, Abdel-Bakky MS, Doberstein K, Schramme A, Beckmann J, Schaefer L et al. CXCL16 and oxLDL are induced in the onset of diabetic nephropathy. J Cell Mol Med. 2009. 13(9B): 3809-25.

[14] Surprenant A, North RA. Signalling at purinergic P2X receptors. Annu Rev Physiol. 2009. 71: 333-59.

[15] Lister MF, Sharkey J, Sawatzky DA, Hodgkiss JP, Davidson DJ, Rossi AG et al. The role of the purinergic P2X7 receptor in inflammation. J Inflamm (Lond). 2007. 4: 5

[16] Idzko M, Ferrari D, Eltzschig HK. Nucleotide signalling during inflammation. Nature. 2014. 509(7500): 310-7.

[17] Beaucage KL, Xiao A, Pollmann SI, Grol MW, Beach RJ, Holdsworth DW et al. Loss of P2X7 nucleotide receptor function leads to abnormal fat distribution in mice. Purinergic Signal. 2014. 10(2): 291-304.

[18] Pupovac A, Foster CM, Sluyter R. Human P2X7 receptor activation induces the rapid shedding of CXCL16. Biochem Biophys Res Commun. 2013. 432(4): 626-31.

[19] Ma KL, Ruan XZ, Powis SH, Moorhead JF, Varghese Z. Anti-atherosclerotic effects of sirolimus on human vascular smooth muscle cells. Am J Physiol Heart Circ Physiol. 2007. 292(6): H2721-8.

[20] Ruan XZ, Moorhead JF, Tao JL, Ma KL, Wheeler DC, Powis SH et al. Mechanisms of dysregulation of low-density lipoprotein receptor expression in vascular smooth muscle cells by inflammatory cytokines. Arterioscler Thromb Vasc Biol. 2006. 26(5): 1150-5.

[21] Ma KL, Ruan XZ, Powis SH, Chen Y, Moorhead JF, Varghese Z. Inflammatory stress exacerbates lipid accumulation in hepatic cells and fatty livers of apolipoprotein E knockout mice. Hepatology. 2008. 48(3): 770-81.

[22] Ma KL, Liu J, Gao M, Wang CX, Ni J, Zhang Y et al. Activation of mTOR contributes to foam cell formation in the radial arteries of patients with end-stage renal disease. Clin Nephrol. 2014. 81(6): 396-404.

[23] Galkina E, Harry BL, Ludwig A, Liehn EA, Sanders JM, Bruce A et al. CXCR6 promotes atherosclerosis by supporting T-cell homing, interferon-gamma production, and macrophage accumulation in the aortic wall. Circulation. 2007. 116(16): 1801-11.

[24] Aslanian AM, Charo IF. Targeted disruption of the scavenger receptor and chemokine CXCL16 accelerates atherosclerosis. Circulation. 2006. 114(6): 583-90.

[25] Sheikine Y, Sirsjo A. CXCL16/SR-PSOX--a friend or a foe in atherosclerosis. Atherosclerosis. 2008. 197(2): 487-95.

[26] Piscopiello M, Sessa M, Anzalone N, Castellano R, Maisano F, Ferrero E et al. P2X7 receptor is expressed in human vessels and might play a role in atherosclerosis. Int J Cardiol. 2013. 168(3):2863-6.

[27] Peng K, Liu L, Wei D, Lv Y, Wang G, Xiong W et al. P2X7R is involved in the progression of atherosclerosis by promoting NLRP3 inflammasome activation. Int J Mol Med. 2015. 35(5): 1179-88. 